\title{
Runge-Kutta method, finding the drowning point when search for crashed planes on the seabed
}

\author{
Haowen Zhong \\ School of North China Electric Power University (Baoding), Baoding 071001, China; \\ Email:495106868@qq.com
}

Keywords: Runge-Kutta method, gray-scale processing , crashed airplane

Abstract. In order to search for crashed planes, we discuss that the resistance changes with velocity of the airplane. Then we draw the settlement trajectory without effects of ocean currents by using Runge-Kutta method. We also do gray-scale processing to seabed topography map around the drowning point to get a group of seabed terrain data, so that we can find the location of the airplane on the seabed.

\section{Introduction}

Gas and liquid are all fluid. The resistance that fluid exerts to moving objects is mainly viscous resistance, pressure drag and wave resistance. In liquids, when an object is in a speed no more than 40 $\mathrm{m} / \mathrm{s}$, it suffers mainly viscous resistance; when its speed is over $40 \mathrm{~m} / \mathrm{s}$, the pressure drag has more effects.

\section{Model Establishment}

To describe the trajectory after the plane loses its power, firstly we need to do stress analysis of the airplane.

Considering the air-stream and according to the analysis, the airplane suffer lift force F, stress drag f and gravity G.

By Newton's second law, is

$$
\left\{\begin{array}{l}
M a_{x}=-f \cos \theta \\
M a_{y}=F+f \sin \theta-M g
\end{array}\right.
$$

To study the settlement of the airplane, we set the drowning point as the coordinate origin. We set the vertical direction as the vertical axis and the direction the airplane move in as the horizontal axis. Here is the Cartesian coordinate system.

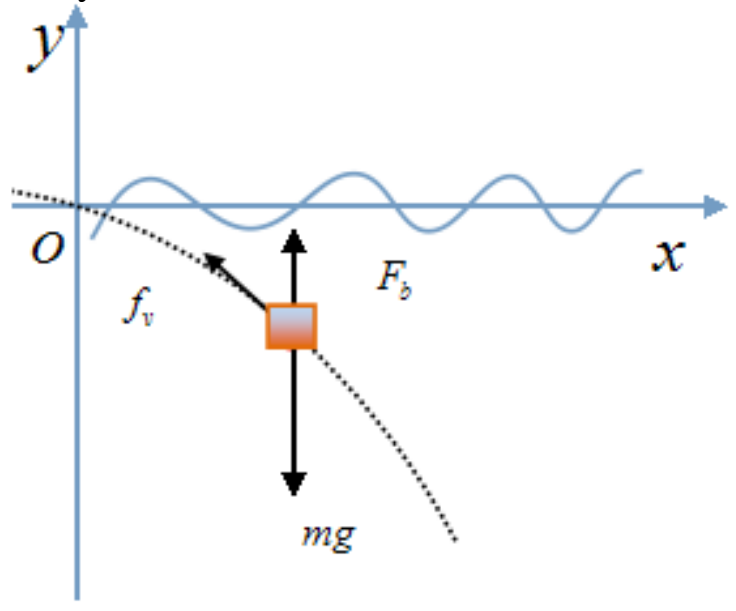

$f_{v}$ is opposite to the direction of settlement velocity. $F_{b}$ is straight up and $m g$ is straight down. 
Make orthogonal decomposition of $f_{v}$ and we get force components in the horizontal and vertical direction as $f_{1}$ and $f_{1}$.We set acceleration of the airplane in the horizontal and vertical direction as $a_{x}{ }^{\prime}$ and ${ }^{a_{y}{ }^{\prime} \text {. }}$.

According to Newton's second law, we get:

$$
\left\{\begin{array}{c}
m a_{x}^{\prime}=-f_{1} \\
m a_{y}^{\prime}=-F_{b}-f_{2}+m g
\end{array}\right.
$$

The main resistance is different when the velocity is different, so we need to do segmented discussion over the velocity of the airplane:

When $v_{b}>40 \mathrm{~m} / \mathrm{s}$, calculated by the viscous drag Formula [1],

$$
f_{v}=k v_{b}
$$

When $v_{b} \leq 40 \mathrm{~m} / \mathrm{s}$, calculated from the pressure drag Formula [1],

$$
f_{v}=\frac{1}{2} \rho_{l} s C_{l} v_{b}^{2}
$$

When the airplane is settling, it soaks completely in the seawater, Buoyancy is calculated as:

$$
F_{b}=\rho_{l} g V
$$

And now we can get two differential equations:

(1) $v_{b}>40 \mathrm{~m} / \mathrm{s}$

$$
\left\{\begin{array}{l}
\frac{d^{2} x}{d t^{2}}=-\frac{\rho_{l} s C_{l}}{2 m}\left(\frac{d x}{d t}\right)^{2} \\
\frac{d^{2} y}{d t^{2}}=-\frac{\rho_{l} s C_{l}}{2 m}\left(\frac{d y}{d t}\right)^{2}-\frac{\rho_{l} g V}{m}+g
\end{array}\right.
$$

(2) $v_{b} \leq 40 \mathrm{~m} / \mathrm{s}$

$$
\left\{\begin{array}{l}
\frac{d^{2} x}{d t^{2}}=-k \frac{d x}{d t} \\
\frac{d^{2} y}{d t^{2}}=-k \frac{d y}{d t}-\frac{\rho_{l} g V}{m}+g
\end{array}\right.
$$

It means that we make a differential equation model to describe the settlement process of the airplane. Known from model one that when the airplane falls into the water its velocity is, namely initial conditions are:

$$
\left\{\begin{array}{l}
x(0)=0 \\
y(0)=0 \\
v_{b}(0)=174
\end{array}\right.
$$

\section{Model Solution}

Firstly we need to obtain the value of the relevant parameters in equation (6)

By assumption, the airplane moves as a triangle with the length l, width $\mathrm{w}$, height $\mathrm{h}$ (unit: $\mathrm{m}$ ) in the seawater. We set MH370 as an example. Here are the relevant parameters after equivalent:

\begin{tabular}{|c|c|c|c|c|c|}
\hline$M$ & $l$ & $w$ & $h$ & $C_{l}$ & $k$ \\
\hline 297550 & 31.5 & 30.2 & 9.25 & 3 & 0.12 \\
\hline
\end{tabular}


We consider the average value of the total area of the airplane as the average force area, and then we get the $\mathrm{S}$. We take the average force area $\mathrm{S}$ and the data in the table above so that the model can be solved.

\subsection{The Solution to the Settlement Locus with the Effects of Ocean Currents out of Consideration}

With the settlement locus obtained and the seabed topography map we founded, we only need to determine the seabed topography curve so that the terminal location of the airplane on the seabed can be available. The last drowning point is where the locus curve and the seabed topography curve get crossed. Further we can judge the area.

According to the seabed topography map we found, we graying it and we extract a list of data of the seabed topography. The seabed topography map can be drawn after Lagrange Interpolation. Here is the image:

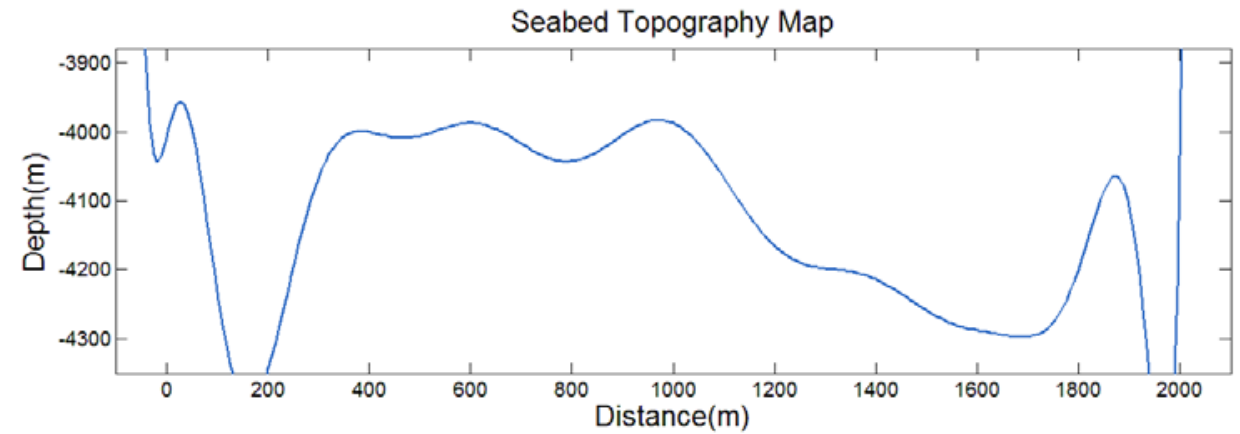

And then draw the trajectories and seabed topography curve within the same coordinate system. The last location of the airplane can be seen.

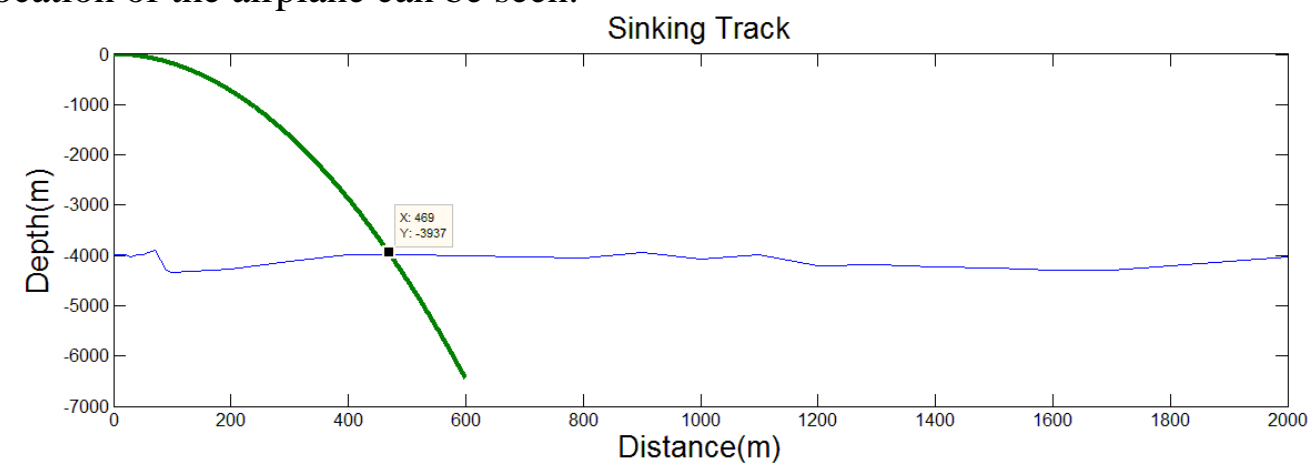

The coordinate of the node is (472.4, -3995), that's the position of the airplane on the seabed.

\section{Summary}

In the case not to consider ocean currents, we do gray-scale processing to seabed topography map around the most possible falling point. At the same time we establish the differential equation model of the settlement process of the airplane. Then draw terrain curve using interpolation in MATLAB. At last we get the most possible location of the airplane.

In practice, parameters related to airplane need to be changed due to different airplane types. The former example is Boeing 777-200(Malaysia Airlines MH370). Now we will replace it with Boeing 747-100 and do comparative analysis.

Parameters of Boeing 747-100 of the parameters are as follow:

\begin{tabular}{|c|c|c|c|}
\hline$M$ & $l$ & $w$ & $h$ \\
\hline 333390 & 35.3 & 29.8 & 9.78 \\
\hline
\end{tabular}

The result is shown below: 


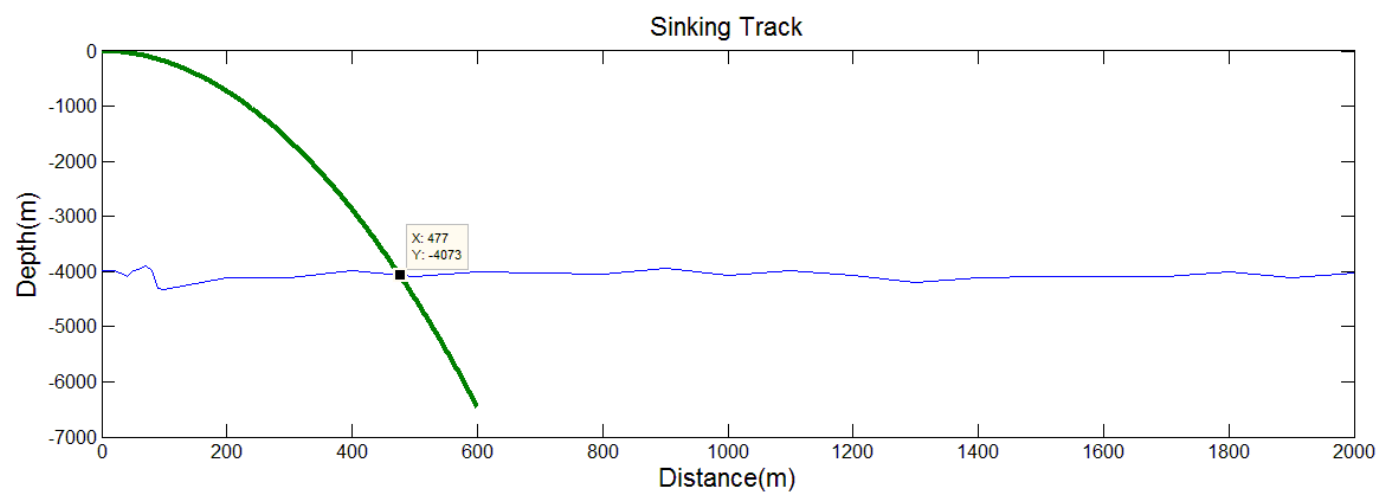

\section{References}

[1]. Mingyuan Zhang, Hydro-mechanics,Beijing: HIGHER EDUCATION PRESS, 2010.

[2]. Resistance, Wikipedia, http://zh.wikipedia.org/wiki/Wikipedia, 2015-02-08.

[3]. airplane knowledge and airplane science curriculum, http:///www.360doc.c, 2015-02-08. 